Article

\title{
Tumor Glucose Metabolism and Its Heterogeneity on F-18 FDG PET/CT Provide Better Prognostication in Nonmetastatic Human Papillomavirus-Related Oropharyngeal Squamous Cell Carcinoma
}

\author{
Hojin Cho $\mathbb{B}$, Soyoung Kim, Kwanhyeong Jo, Yong Hyu Jeong $(\mathbb{D}$ and Won Jun Kang *
}

Citation: Cho, H.; Kim, S.; Jo, K.; Jeong, Y.H.; Kang, W.J. Tumor Glucose Metabolism and Its Heterogeneity on F-18 FDG PET/CT Provide Better Prognostication in

Nonmetastatic Human

Papillomavirus-Related Oropharyngeal Squamous Cell Carcinoma. Cancers 2021, 13, 5538. https://doi.org/10.3390/ cancers 13215538

Academic Editor: David Wong

Received: 21 October 2021

Accepted: 3 November 2021

Published: 4 November 2021

Publisher's Note: MDPI stays neutral with regard to jurisdictional claims in published maps and institutional affiliations.

Copyright: (C) 2021 by the authors Licensee MDPI, Basel, Switzerland. This article is an open access article distributed under the terms and conditions of the Creative Commons Attribution (CC BY) license (https:/ / creativecommons.org/licenses/by/ $4.0 /)$.
Department of Nuclear Medicine, Severance Hospital, Yonsei University College of Medicine, Seoul 03722, Korea; hojincho@yuhs.ac (H.C.); saranghayo3@yuhs.ac (S.K.); phe_ea@naver.com (K.J.); fulif@yuhs.ac (Y.H.J.)

* Correspondence: mdkwj@yuhs.ac

Simple Summary: Human papillomavirus (HPV)-related oropharyngeal squamous cell carcinoma (OPSCC) emerged as a distinct disease with a favorable prognosis, and a separate staging system was introduced. However, a subset of patients harbor a poor prognosis. We aimed to evaluate the prognostic role of metabolic parameters on baseline F-18 FDG PET/CT in patients with HPVrelated OPSCC. We retrospectively reviewed patients who were diagnosed with stage I, II, and III HPV-related OPSCC using the 8th TNM staging. Metabolic features on baseline F-18 FDG PET/CT, such as higher tumor glucose metabolism derived from tumor $\mathrm{SUV}_{\max }$ to liver $\mathrm{SUV}_{\text {mean }}$ ratio, and increased intratumoral heterogeneity inferred from coefficient of variation were associated with poorer progression-free survival and overall survival. Further study is warranted to address the possible implications of F-18 FDG PET/CT on treatment de-intensification in these patients.

Abstract: Background: We aimed to evaluate the prognostic role of metabolic parameters on baseline F-18 fluorodeoxyglucose (FDG) PET/CT in patients with human papillomavirus (HPV)-related oropharyngeal squamous cell carcinoma (OPSCC). Methods: We retrospectively reviewed patients who were diagnosed with nonmetastatic HPV-related OPSCC using the 8th TNM staging system from 2010 to 2015 and underwent baseline F-18 FDG PET/CT. Tumor SUV max to liver SUV mean ratio $\left(\mathrm{SUV}_{\max }-\mathrm{TLR}\right)$, metabolic tumor volume (MTV), tumor total lesion glycolysis to liver $\mathrm{SUV}_{\text {mean }}$ ratio (TLG-TLR), and coefficient of variation (CV) of the primary tumor were measured. Patients were primarily treated with surgery or radiotherapy. Endpoints were progression-free survival (PFS) and overall survival (OS). Results: Ninety consecutive patients (male, 72; female, 18) were enrolled. They were followed up for a median of 77.4 months (interquartile range, 48.4-106.4). Sixteen patients progressed, and 13 died. Multivariate analysis revealed that patients with advanced age, overall stage, and higher SUV max $-\mathrm{TLR}$ or CV had poorer PFS and OS. Conclusion: Higher $\mathrm{SUV}_{\max }-\mathrm{TLR}$ and CV of the primary tumor on baseline F-18 FDG PET/CT were associated with poorer PFS and OS in patients with nonmetastatic HPV-related OPSCC. Further study is warranted to address the possible implications of F-18 FDG PET/CT on treatment de-intensification in these patients.

Keywords: oropharyngeal carcinoma; prognosis; FDG; PET/CT

\section{Introduction}

The incidence of oropharyngeal squamous cell carcinoma (OPSCC) is increasing, with more than 90,000 new cases each year globally [1]. Human papillomavirus (HPV) infection results in an increased incidence of oropharyngeal cancer, and recent studies have shown that approximately 70\% of OPSCC cases are associated with HPV in North America and Europe [2-6]. HPV-related OPSCC is clinically and biologically different from HPVunrelated OPSCC [7-9]. HPV-related OPSCC generally occurs in a younger and healthier 
population with less exposure to tobacco smoke, and it has better overall survival (OS) and progression-free survival (PFS) $[10,11]$. The 8 th edition of the American Joint Committee on Cancer staging system provides a better understanding of the clinical behavior and tumor biology, and a separate staging algorithm was introduced for HPV-related OPSCC, resembling the $\mathrm{N}$ classification of nasopharyngeal carcinoma [12,13]. Approximately $80 \%$ of patients with HPV-related OPSCC will probably be cured [10,14]. However, a subset of patients have a poor prognosis, and this population needs to be investigated.

Patients with early stage OPSCC may be treated with surgery or radiotherapy $[15,16]$. For patients with more advanced stages, concurrent cisplatin-based chemoradiotherapy is administered either definitely or as postoperative adjuvant treatment $[17,18]$. The current treatments are strict and cause substantial acute and late toxicity [19]. Because patients with HPV-related OPSCC have a better prognosis, a growing body of research has been focused on treatment de-intensification maintaining the current cure rates while reducing treatmentrelated morbidities. Although a subset of HPV-related OPSCC show a good prognosis, the early results from treatment de-intensification trials support to maintain current treatment guidelines $[20,21]$. Therefore, precisely identifying this good-risk population is crucial.

It is not clear that F-18 fluorodeoxyglucose (FDG) positron emission tomography/ computed tomography (PET/CT) can reclassify patients with HPV-related OPSCC into different risk categories. F-18 FDG PET/CT has been widely used in staging and evaluating therapy response in patients with HPV-related OPSCC [22-24]. Previous studies also showed that higher maximum standardized uptake value $\left(\mathrm{SUV}_{\max }\right)$, metabolic tumor volume (MTV), and total lesion glycolysis (TLG) are associated with worse prognosis $[25,26]$. By contrast, some studies reported that the metabolic parameters on F-18 FDG PET/CT do not predict patients' outcome $[27,28]$. These studies were based on the 7 th TNM staging system, and the prognostic role of F-18 FDG PET/CT has not been validated using the 8 th TNM staging system. The present study aimed to determine whether metabolic parameters on baseline F-18 FDG PET/CT improve prognostication in patients with HPV-related OPSCC using the current 8th TNM staging system.

\section{Materials and Methods}

\subsection{Patients}

We retrospectively reviewed the medical records of patients who were newly diagnosed with stage I, II, and III HPV-related OPSCC using the 8th TNM staging system and underwent F-18 FDG PET/CT scan from 2010 to 2015. The HPV status was determined using p16 immunohistochemistry. Patients with a history of other malignancies and who received any treatment prior to F-18 FDG PET/CT scans were excluded. Patients were reviewed at a multidisciplinary tumor board to determine primary treatment. In patients who received surgery as the primary treatment, postoperative radiation therapy was given to patients with pT3-4 disease, a close margin, one positive node greater than $3 \mathrm{~cm}$ or multiple positive nodes, lymphovascular invasion, or perineural invasion. Postoperative chemoradiotherapy was delivered to patients with extranodal extension or a positive margin.

After treatment completion, patients were followed up every 3 months for the first 2 years, then every 6 months until 5 years, and annually thereafter. Treatment response and follow-up evaluation were assessed by recording their history and performing a physical examination, neck CT, and/or MRI. F-18 FDG PET/CT, whole-body bone scan, or chest $\mathrm{CT}$ were obtained according to the referring physicians' preference; follow-up imaging was performed every 3-6 months for the first 2 years and annually thereafter. Treatment response was assessed with the response evaluation criteria in solid tumors (RECIST), version 1.1.

\subsection{F-18 FDG PET/CT}

All patients underwent F-18 FDG PET/CT on either a Biograph TruePoint 40 PET/CT scanner (Siemens Healthcare, Erlangen, Germany) or a Discovery STe PET/CT scanner 
(GE Healthcare, Milwaukee, WI, USA). Patients fasted for at least $6 \mathrm{~h}$ before the scan, and peripheral blood glucose levels were no higher than $140 \mathrm{mg} / \mathrm{dL}$ before F-18 FDG injection. Approximately 5.5 MBq of F-18 FDG per kg of body weight was administered intravenously $1 \mathrm{~h}$ before the start of imaging. After the initial low-dose CT (Biograph TruePoint 40: $36 \mathrm{~mA}, 120 \mathrm{kVp}$; Discovery STe: $30 \mathrm{~mA}, 140 \mathrm{kVp}$ ), standard PET imaging was conducted from the cerebellum to the mid-thigh, with acquisition times of $2.5 \mathrm{~min} / \mathrm{bed}$ position for the Biograph TruePoint 40 scanner and $3 \mathrm{~min} /$ bed position for the Discovery STe scanner in a three-dimensional mode. PET images were reconstructed iteratively with CT-based attenuation correction.

\subsection{Image Analysis}

All F-18 FDG PET/CT images were reviewed by two nuclear medicine physicians using MIM imaging software (MIM 6.8; MIM Software Inc., Cleveland, OH, USA). The $\mathrm{SUV}_{\text {max }}$ values were measured in a volume of interest (VOI) drawn on PET images. In each patient, the SUV max, MTV (the metabolically active volume of the tumor), TLG (the product of mean SUV and MTV), and coefficient of variation (CV; the ratio of the standard deviation to the mean) of the primary tumor were measured. Normal background liver SUV mean and standard deviation (SD) were measured by drawing a 3-cm-sized spherical VOI in the right lobe of the liver. To calculate the MTV threshold, we used the following formula: liver $\mathrm{SUV}_{\text {mean }}+\left(2 \times\right.$ liver $\left.\mathrm{SUV}_{\mathrm{SD}}\right)$ [29]. The SUV of the VOI was calculated as follows: decay-corrected activity (kilobecquerel) per mm of tissue volume/injected F-18 FDG activity (kilobecquerel)/body weight (g). The SUV $\mathrm{max}_{\text {and }}$ TLG of the primary tumor were divided by mean liver SUV to mitigate variability in SUV measurements, yielding tumor $S U V_{\max }$ to liver $S U V_{\text {mean }}$ ratio $\left(S U V_{\max }-\mathrm{TLR}\right)$, and tumor total lesion glycolysis to liver $S U V_{\text {mean }}$ ratio (TLG-TLR), respectively.

\subsection{Statistical Analysis}

The following variables were included in univariate analyses: age, sex, ECOG performance status, stage, smoking history, primary treatment, and metabolic parameters on F-18 FDG PET/CT (SUV max $-T L R, M T V$, TLG-TLR, and CV of the primary tumor). Survival time was calculated from the date of diagnosis to the date of development of first progression or death from any cause. We used a Cox proportional-hazards model to evaluate PFS and OS. Statistical analyses were performed using R software (version 4.1.0, R Core Team, 2021, Vienna, Austria), and a $p$ value $<0.05$ was considered statistically significant. The optimal cutoffs for continuous variables were calculated using the maximally selected rank statistics [30].

\section{Results}

\subsection{Patient Characteristics}

A total of 90 consecutive patients (72 males and 18 females) were enrolled in this study. Their mean age was 57.3 (SD, 8.6) years. Among the 90 patients, 61 (67.8\%), $11(12.2 \%)$, and $18(20.0 \%)$ had stage I, II, and III HPV-related OPSCC, respectively. Fifty-two patients $(57.8 \%)$ had a smoking history equal to or less than 10 pack-years. Seventy-seven patients $(85.6 \%)$ underwent surgery as primary treatment. Among them, 71 (92.2\%) received adjuvant radiotherapy $(n=15)$ or chemoradiotherapy $(n=56)$. Chemotherapy was given to 76 patients $(84.4 \%)$. (Table 1$)$. The patients were followed up for a median of 77.4 months (interquartile range, 48.4 to 106.4). During the follow-up period, 16 (17.8\%) had progressive disease, and $13(14.4 \%)$ died. The two-year PFS and OS rates were $84.4 \%$ and 95.6\%, respectively (Figure 1). Significant correlations were found between $\mathrm{SUV}_{\max }-\mathrm{TLR}, \mathrm{MTV}$, TLG-TLR, and CV $(p<0.001)$. Therefore, only one of the metabolic parameters on F-18 FDG PET/CT was included in the multivariate analysis at a time. 
Table 1. Patient characteristics.

\begin{tabular}{|c|c|}
\hline Characteristic & All Patients $(n=90)$ \\
\hline Age, mean $\pm S D$, years & $57.3 \pm 8.6$ \\
\hline \multicolumn{2}{|l|}{ Sex, $n(\%)$} \\
\hline Male & $72(80.0)$ \\
\hline Female & $18(20.0)$ \\
\hline \multicolumn{2}{|l|}{ ECOG, $n(\%)$} \\
\hline 0 & $33(36.7)$ \\
\hline 1 & $57(63.3)$ \\
\hline \multicolumn{2}{|l|}{ Overall stage, $n(\%)$} \\
\hline Stage I & $61(67.8)$ \\
\hline Stage II & $11(12.2)$ \\
\hline Stage III & $18(20.0)$ \\
\hline \multicolumn{2}{|l|}{ Tumor stage, $n(\%)$} \\
\hline $\mathrm{T} 1$ & $19(21.1)$ \\
\hline $\mathrm{T} 2$ & $45(50.0)$ \\
\hline T3 & $8(8.9)$ \\
\hline $\mathrm{T} 4$ & $18(20.0)$ \\
\hline \multicolumn{2}{|l|}{ Nodal stage, $n(\%)$} \\
\hline No & $9(10.0)$ \\
\hline N1 & $68(75.6)$ \\
\hline N2 & $12(13.3)$ \\
\hline N3 & $1(1.1)$ \\
\hline \multicolumn{2}{|l|}{ Smoking history, $n(\%)$} \\
\hline Never or $\leq 10$ pack-years & $52(57.8)$ \\
\hline$>10$ pack-years & $38(42.2)$ \\
\hline \multicolumn{2}{|l|}{ Primary treatment, $n(\%)$} \\
\hline Surgery & $77(85.6)$ \\
\hline \multicolumn{2}{|l|}{ Surgical approach, $n(\%)$} \\
\hline Open & $37(48.1)$ \\
\hline Robotic & $40(51.9)$ \\
\hline \multicolumn{2}{|l|}{ Resection margin, $n(\%)$} \\
\hline Negative & $52(67.5)$ \\
\hline Positive & $25(32.5)$ \\
\hline \multicolumn{2}{|l|}{ Extracapsular spread, $n(\%)$} \\
\hline Negative & $33(42.9)$ \\
\hline Positive & $44(57.1)$ \\
\hline \multicolumn{2}{|c|}{ Lymphovascular invasion, $n(\%)$} \\
\hline Negative & $52(67.5)$ \\
\hline Positive & $25(32.5)$ \\
\hline \multicolumn{2}{|l|}{ Perineural invasion, $n(\%)$} \\
\hline Negative & $66(85.7)$ \\
\hline Positive & $11(14.3)$ \\
\hline \multicolumn{2}{|l|}{ Adjuvant therapy, $n(\%)$} \\
\hline None & $6(7.8)$ \\
\hline Radiotherapy & $15(19.5)$ \\
\hline Chemoradiotherapy & $56(72.7)$ \\
\hline Radiotherapy & $13(14.4)$ \\
\hline \multicolumn{2}{|l|}{ Chemotherapy, $n$ (\%) } \\
\hline Yes & $76(84.4)$ \\
\hline No & $14(15.6)$ \\
\hline $\mathrm{SUV}_{\max }-\mathrm{TLR}$, median (range) & $5.4(1.5-11.0)$ \\
\hline MTV, median (range), mL & $7.9(0.3-61.5)$ \\
\hline TLG-TLR, median (range) & $26.4(1.3-243.7)$ \\
\hline $\mathrm{CV}$, mean $\pm \mathrm{SD}, \%$ & $27.8 \pm 6.3$ \\
\hline
\end{tabular}

$\mathrm{SUV}_{\max }-\mathrm{TLR}$, tumor SUV $\max$ to liver $\mathrm{SUV}_{\text {mean }}$ ratio; MTV, metabolic tumor volume; TLG-TLR, tumor total lesion glycolysis to liver $\mathrm{SUV}_{\text {mean }}$ ratio; $\mathrm{CV}$, coefficient of variation. 


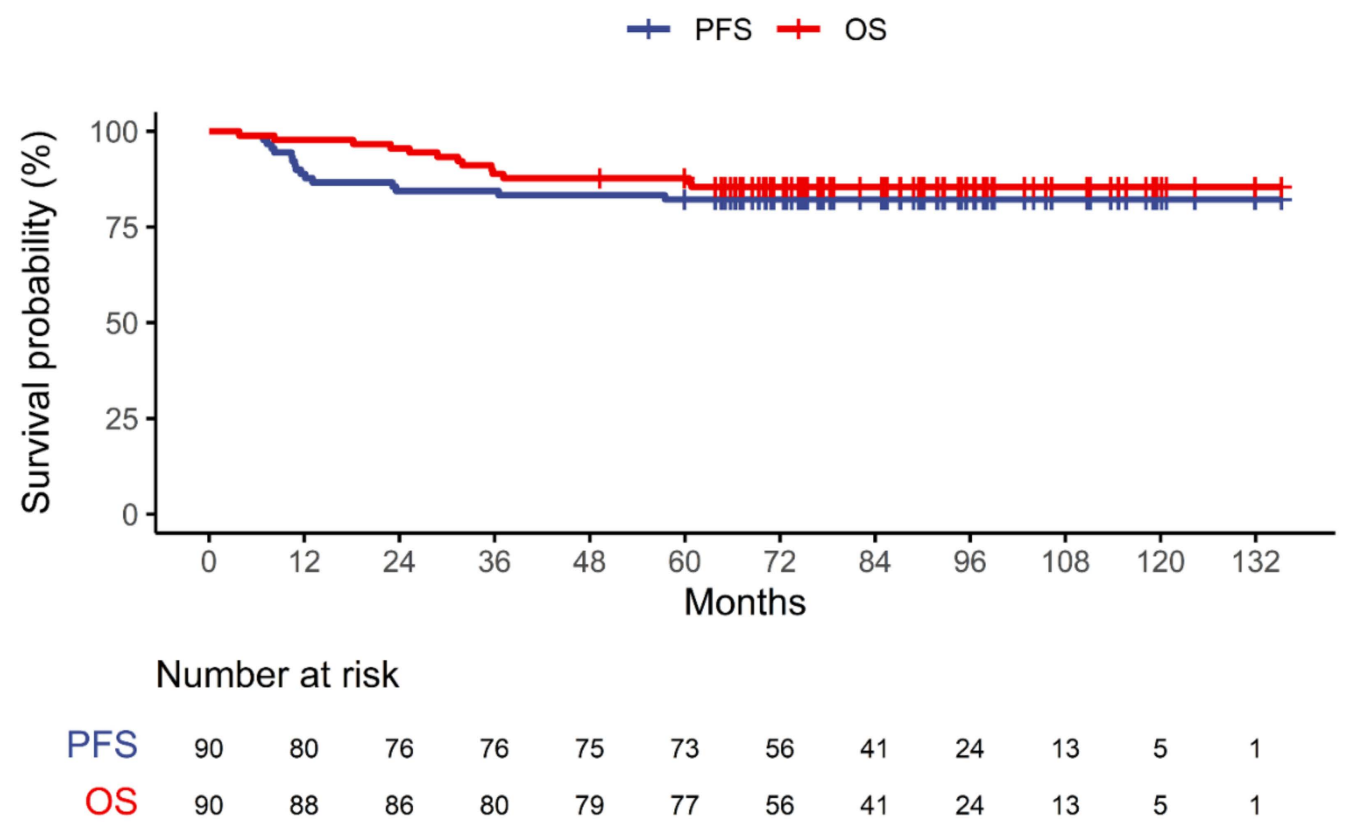

Figure 1. Progression-free survival (PFS) and overall survival (OS) during the follow-up period. Tick marks represent censored data.

\subsection{Prognostic Values of Clinical Parameters and Metabolic Parameters of F-18 FDG PET/CT}

The optimal cutoff values for age, $\mathrm{SUV}_{\max }-\mathrm{TLR}, \mathrm{MTV}$, TLG-TLR, and CV in predicting PFS were 57, 4.8, 17.1, 84.2, and 27.9, respectively. Poorer PFS was associated with advanced age $(\leq 57(n=45(50.0 \%))$ vs. $>57(n=45(50.0 \%)$ years $) ; p=0.04$, hazard ratio $(\mathrm{HR})=3.21)$, higher overall stage (I-II vs. III; $p<0.001, \mathrm{HR}=7.21$ ), radiotherapy as primary treatment, as compared with surgery $(p=0.004, \mathrm{HR}=4.37), \operatorname{SUV}_{\max }-\mathrm{TLR}(\leq 4.8(n=35(38.9 \%))$ vs. $>4.8(n=55(61.1 \%)) ; p=0.02, \mathrm{HR}=11.03)$, MTV $(\leq 17.1(n=75(83.3 \%))$ vs. $>17.1 \mathrm{~mL}$ $(n=15(16.7 \%)) ; p<0.001, \mathrm{HR}=6.81)$, TLG-TLR $(\leq 84.2(n=81(90.0 \%))$ vs. $>84.2(n=9$ $(10.0 \%)) ; p<0.001, \mathrm{HR}=8.34)$, and CV $(\leq 27.9(n=43(47.8 \%))$ vs. $>27.9 \%(n=47(52.2 \%))$; $p=0.007, \mathrm{HR}=16.54)$. Nodal stage did not affect PFS among patients with same overall stage (all $p$ values were greater than 0.05 ).

Two multivariate models showed significant associations between metabolic parameters and PFS. The first model (PFS model 1) included age, overall stage, primary treatment, and SUV $V_{\max }$-TLR; advanced age, higher overall stage, and $\mathrm{SUV}_{\max }$-TLR remained significant in predicting worse PFS. The second model (PFS model 4) included age, overall stage, primary treatment, and CV; advanced age, higher overall stage, and CV were significant predictors of poorer PFS (Table 2).

Table 2. Univariate and multivariate analyses for progression-free survival.

\begin{tabular}{|c|c|c|}
\hline Characteristic & Hazard Ratio (95\% CI) & $p^{*}$ \\
\hline \multicolumn{3}{|c|}{ Univariate Analysis } \\
\hline Age & & 0.04 \\
\hline $\begin{array}{l}\leq 57 \text { years } \\
>57 \text { years }\end{array}$ & $3.21(1.03-9.95)$ & \\
\hline Sex & & 0.44 \\
\hline Female & $0.56(0.13-2.45)$ & \\
\hline $\begin{array}{c}\text { ECOG } \\
0\end{array}$ & & 0.13 \\
\hline 1 & $2.65(0.75-9.30)$ & \\
\hline $\begin{array}{l}\text { Smoking history } \\
\text { Never or } \leq 10 \text { pack-years }\end{array}$ & & 0.24 \\
\hline$>10$ pack-years & $1.80(0.67-4.84)$ & \\
\hline
\end{tabular}


Table 2. Cont.

\begin{tabular}{|c|c|c|}
\hline Characteristic & Hazard Ratio (95\% CI) & $p^{*}$ \\
\hline \multicolumn{3}{|l|}{ I-II } \\
\hline III & 7.21 (2.67-19.45) & \\
\hline \multicolumn{3}{|l|}{ Surgery } \\
\hline Radiotherapy & 4.37 (1.58-12.07) & \\
\hline Chemotherapy & & 0.73 \\
\hline $\begin{array}{l}\text { Yes } \\
\text { No }\end{array}$ & $1.30(0.29-5.70)$ & \\
\hline $\begin{array}{c}\mathrm{SUV}_{\max }-\mathrm{TLR} \\
\leq 4.8\end{array}$ & & 0.02 \\
\hline$>4.8$ & $11.03(1.46-83.53)$ & \\
\hline \multicolumn{3}{|l|}{$\leq 17.1 \mathrm{~mL}$} \\
\hline$>17.1 \mathrm{~mL}$ & $6.81(2.54-18.27)$ & \\
\hline $\begin{array}{l}\text { TLG-TLR } \\
<84.2\end{array}$ & & $<0.001$ \\
\hline$>84.2$ & $8.34(2.97-23.45)$ & \\
\hline $\mathrm{CV}$ & & 0.007 \\
\hline \multicolumn{3}{|l|}{$\leq 27.9 \%$} \\
\hline \multicolumn{3}{|c|}{ Multivariate analysis including age, overall stage, $\mathrm{SUV}_{\max }-\mathrm{TLR}$, and primary treatment (PFS model 1) } \\
\hline Age ( $\leq 57$ vs. $>57$ years $)$ & $4.08(1.29-12.93)$ & 0.02 \\
\hline Overall stage (I-II vs. III) & $5.36(1.69-17.03)$ & 0.004 \\
\hline Primary treatment (surgery vs. radiotherapy) & $1.78(0.53-5.97)$ & 0.35 \\
\hline $\mathrm{SUV}_{\max }-\mathrm{TLR}(\leq 4.8$ vs. $>4.8)$ & $9.92(1.26-77.86)$ & 0.03 \\
\hline \multicolumn{3}{|c|}{ Multivariate analysis including age, overall stage, MTV, and primary treatment (PFS model 2) } \\
\hline Age ( $\leq 57$ vs. $>57$ years $)$ & $3.67(1.16-11.55)$ & 0.03 \\
\hline Overall stage (I-II vs. III) & $3.73(1.15-12.04)$ & 0.03 \\
\hline Primary treatment (surgery vs. radiotherapy) & $1.58(0.43-5.88)$ & 0.49 \\
\hline MTV $(\leq 17.1$ vs. $>17.1 \mathrm{~mL})$ & $3.30(0.90-12.04)$ & 0.07 \\
\hline \multicolumn{3}{|c|}{ Multivariate analysis including age, overall stage, TLG-TLR, and primary treatment (PFS model 3) } \\
\hline Age ( $\leq 57$ vs. $>57$ years $)$ & $4.66(1.39-15.62)$ & 0.01 \\
\hline Overall stage (I-II vs. III) & $3.63(1.13-11.64)$ & 0.03 \\
\hline Primary treatment (surgery vs. radiotherapy) & $1.92(0.56-6.63)$ & 0.30 \\
\hline TLG-TLR $(\leq 84.2$ vs. $>84.2)$ & $3.80(0.94-15.30)$ & 0.06 \\
\hline \multicolumn{3}{|c|}{ Multivariate analysis including age, overall stage, CV, and primary treatment (PFS model 4) } \\
\hline Age ( $\leq 57$ vs. $>57$ years $)$ & $5.13(1.55-16.95)$ & 0.007 \\
\hline Overall stage (I-II vs. III) & $6.62(1.86-23.63)$ & 0.004 \\
\hline Primary treatment (surgery vs. radiotherapy) & $1.29(0.36-4.60)$ & 0.70 \\
\hline $\mathrm{CV}(\leq 27.9$ vs. $>27.9 \%)$ & $16.77(2.05-136.96)$ & 0.009 \\
\hline
\end{tabular}

${ }^{*}$ Wald test. CI, confidence interval; $\mathrm{SUV}_{\max }-\mathrm{TLR}$, tumor $\mathrm{SUV}_{\max }$ to liver $\mathrm{SUV}_{\text {mean }}$ ratio; $\mathrm{MTV}$, metabolic tumor volume; TLG-TLR, tumor total lesion glycolysis to liver $\mathrm{SUV}_{\text {mean }}$ ratio; $\mathrm{CV}$, coefficient of variation; $\mathrm{PFS}$, progression-free survival.

The optimal cutoff values for age, $\mathrm{SUV}_{\max }-\mathrm{TLR}, \mathrm{MTV}$, TLG-TLR, and CV in predicting OS were $57,4.9,17.1,50.0$, and 27.9 , respectively. Advanced age ( $\leq 57 \mathrm{vs.}>57$ years; $p=0.02$, $\mathrm{HR}=5.94$ ), higher overall stage (I-II vs. III; $p<0.001, \mathrm{HR}=8.29)$, radiotherapy as primary treatment $(p=0.001, \mathrm{HR}=6.29)$, higher SUV $\max -\mathrm{TLR}(\leq 4.9(n=39(43.3 \%))$ vs. $>4.9(n=51$ $(56.7 \%)) ; p=0.02, \mathrm{HR}=10.38)$, MTV ( $\leq 17.1$ vs. $>17.1 \mathrm{~mL} ; p=0.002, \mathrm{HR}=5.55)$, TLG-TLR $(\leq 50.0(n=69(76.7 \%))$ vs. $>50.0(n=21(23.3 \%)) ; p=0.001, \mathrm{HR}=6.48)$, and CV $(\leq 27.9$ vs. $>27.9 \% ; p=0.01, \mathrm{HR}=12.64$ ) were associated with worse OS. Nodal stage also did not affect OS among patients with the same overall stage (all $p$ values were greater than 0.05 ).

Significant associations between metabolic parameters and OS were found in two multivariate models. The first model (OS model 1) included age, overall stage, primary 
treatment, and SUV $V_{\max }-\mathrm{TLR}$; advanced age, higher overall stage, and $\mathrm{SUV}_{\max }-\mathrm{TLR}$ remained significant in predicting poorer OS. The second model (OS model 4) included age, overall stage, primary treatment, and CV; advanced age, higher overall stage, and CV were significant predictors of worse OS (Table 3).

Table 3. Univariate and multivariate analyses for overall survival.

\begin{tabular}{|c|c|c|}
\hline Characteristic & Hazard Ratio (95\% CI) & $p^{*}$ \\
\hline \multicolumn{3}{|c|}{ Univariate Analysis } \\
\hline Age & & 0.02 \\
\hline \multicolumn{3}{|l|}{$\leq 57$ years } \\
\hline$>57$ years & $5.94(1.32-26.80)$ & \\
\hline Sex & & 0.66 \\
\hline \multicolumn{3}{|l|}{ Male } \\
\hline Female & $0.71(0.16-3.20)$ & \\
\hline ECOG & & 0.12 \\
\hline \multicolumn{3}{|l|}{0} \\
\hline 1 & $3.32(0.74-14.99)$ & \\
\hline Smoking history & & 0.36 \\
\hline \multicolumn{3}{|l|}{ Never or $\leq 10$ pack-years } \\
\hline$>10$ pack-years & $1.66(0.56-4.94)$ & \\
\hline Overall stage & & $<0.001$ \\
\hline \multicolumn{3}{|l|}{ I-II } \\
\hline III & $8.29(2.70-25.47)$ & \\
\hline Primary treatment & & 0.001 \\
\hline \multicolumn{3}{|l|}{ Surgery } \\
\hline Radiotherapy & $6.29(2.10-18.79)$ & \\
\hline Chemotherapy & & 0.95 \\
\hline Yes & $0.95(0.21-4.31)$ & \\
\hline \multicolumn{3}{|c|}{ 然 } \\
\hline \multicolumn{3}{|l|}{$\leq 4.9$} \\
\hline$>4.9$ & $10.38(1.35-79.89)$ & \\
\hline MTV & & 0.002 \\
\hline \multicolumn{3}{|l|}{$\leq 17.1 \mathrm{~mL}$} \\
\hline TLG-TLR & & 0.001 \\
\hline \multicolumn{3}{|l|}{$\leq 50.0$} \\
\hline$>50.0$ & $6.48(2.12-19.86)$ & \\
\hline $\mathrm{CV}$ & & 0.01 \\
\hline \multicolumn{3}{|l|}{$\leq 27.9 \%$} \\
\hline$>27.9 \%$ & $12.64(1.64-97.24)$ & \\
\hline \multicolumn{3}{|c|}{ Multivariate analysis including age, overall stage, $\mathrm{SUV}_{\max }-\mathrm{TLR}$, and primary treatment (OS model 1) } \\
\hline Age ( $\leq 57$ vs. $>57$ years $)$ & $8.24(1.75-38.85)$ & 0.008 \\
\hline Overall stage (I-II vs. III) & $4.57(1.20-17.38)$ & 0.03 \\
\hline Primary treatment (surgery vs. radiotherapy) & $3.23(0.85-12.27)$ & 0.08 \\
\hline $\operatorname{SUV}_{\max }-\mathrm{TLR}(\leq 4.9$ vs. $>4.9)$ & $10.16(1.29-79.70)$ & 0.03 \\
\hline \multicolumn{3}{|c|}{ Multivariate analysis including age, overall stage, MTV, and primary treatment (OS model 2) } \\
\hline Age ( $\leq 57$ vs. $>57$ years $)$ & $7.55(1.59-35.88)$ & 0.01 \\
\hline Overall stage (I-II vs. III) & $3.99(1.08-14.79)$ & 0.04 \\
\hline Primary treatment (surgery vs. radiotherapy) & $2.83(0.68-11.76)$ & 0.15 \\
\hline $\operatorname{MTV}(\leq 17.1$ vs. $>17.1 \mathrm{~mL})$ & $2.74(0.67-11.13)$ & 0.16 \\
\hline \multicolumn{3}{|c|}{ Multivariate analysis including age, overall stage, TLG-TLR, and primary treatment (OS model 3) } \\
\hline Age ( $\leq 57$ vs. $>57$ years $)$ & $7.32(1.56-34.28)$ & 0.01 \\
\hline Overall stage (I-II vs. III) & $3.51(0.94-13.11)$ & 0.06 \\
\hline Primary treatment (surgery vs. radiotherapy) & $3.29(0.88-12.31)$ & 0.08 \\
\hline TLG-TLR $(\leq 50.0$ vs. $>50.0)$ & $3.28(0.94-11.42)$ & 0.06 \\
\hline
\end{tabular}


Table 3. Cont.

\begin{tabular}{ccc}
\hline Characteristic & Hazard Ratio (95\% CI) & $p^{*}$ \\
\hline Multivariate analysis including age, overall stage, CV, and primary treatment (OS model 4) & 0.008 \\
\hline Age ( $\leq$ 57 vs. $>57$ years) & $8.17(1.73-38.54)$ & 0.02 \\
Overall stage (I-II vs. III) & $5.42(1.25-23.53)$ & 0.31 \\
CV $(\leq 27.9$ vs. $>27.9 \%)$ & $2.11(0.50-8.85)$ & 0.03 \\
\hline
\end{tabular}

${ }^{*}$ Wald test. CI, confidence interval; $\mathrm{SUV}_{\max }-\mathrm{TLR}$, tumor $\mathrm{SUV}_{\max }$ to liver $\mathrm{SUV}_{\text {mean }}$ ratio; $\mathrm{MTV}$, metabolic tumor volume; TLG-TLR, tumor total lesion glycolysis to liver $\mathrm{SUV}_{\text {mean }}$ ratio; $\mathrm{CV}$, coefficient of variation; $\mathrm{OS}$, overall survival.

Kaplan-Meier estimates also reveal that patients with HPV-related OPSCC can be classified into groups with different PFS and OS according to stage, and SUV $\max$-TLR or CV (Figure 2). In stage I and II disease, higher SUV $\max -T L R$ and CV were associated with poorer OS $(p=0.03)$ and PFS $(p=0.02)$, respectively. With higher SUV $\max _{-T L R}$ and $\mathrm{CV}$, stage III disease showed poorer PFS and OS than stage I and II disease $(p<0.001)$, respectively. Subgroup analyses in patients primarily treated with surgery also showed that metabolic parameters along with overall stage and lymphovascular invasion predicted

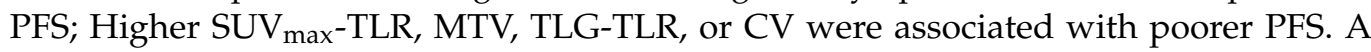
multivariate model with overall stage, lymphovascular invasion, and CV did not reach statistical significance to predict overall survival, but showed a trend toward shorter overall survival with higher CV. (Tables 4-6). Two representative cases with different outcomes are shown in Figure 3. Although the patients had the same stage of disease, higher $\mathrm{SUV}_{\max }$ TLR and CV predicted poorer PFS and OS. Figure 4 demonstrates the predictive nomogram estimated for the 1- and 2-year progression-free and overall survival rates based on the selected parameters in the multivariate Cox proportional hazards models.

A

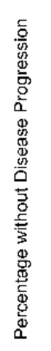

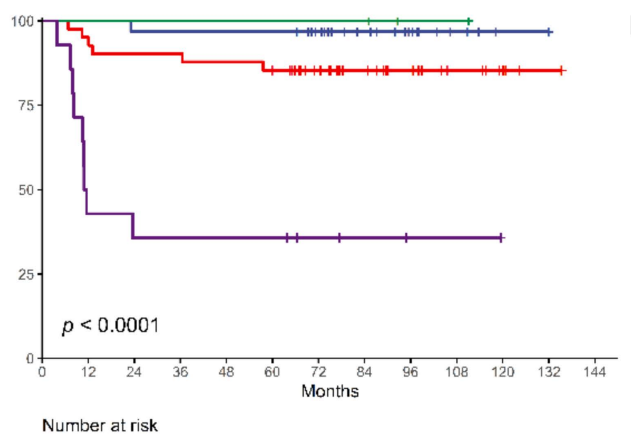

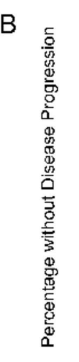
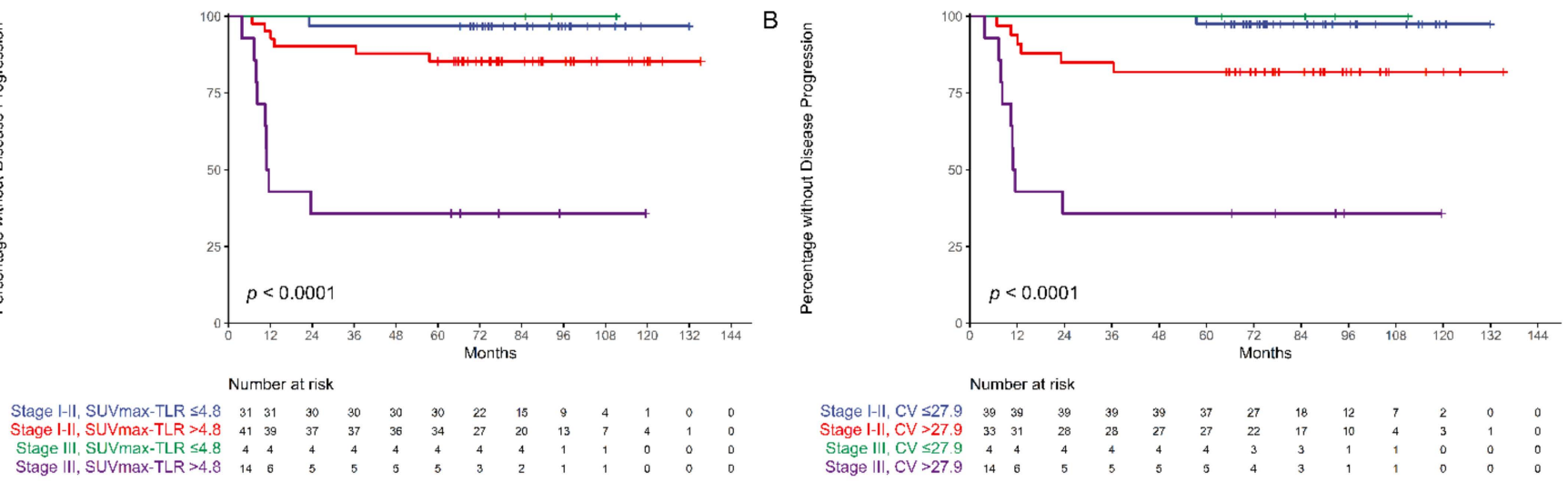

C

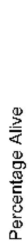

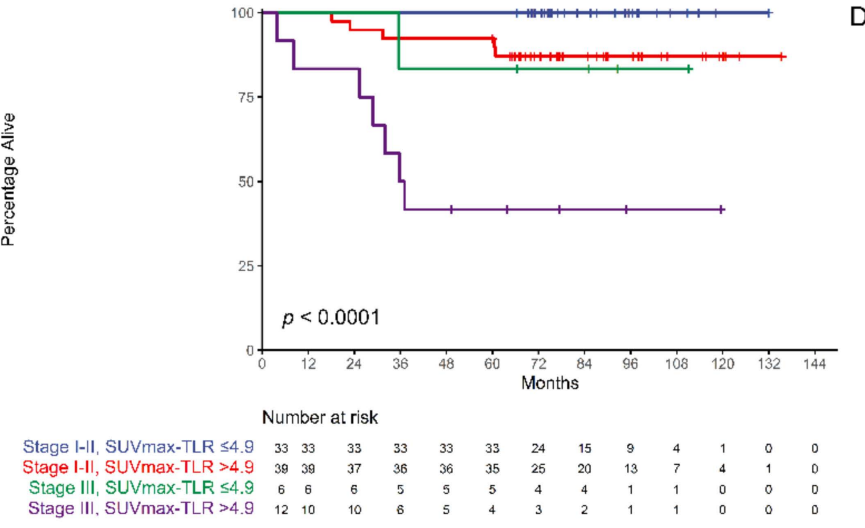

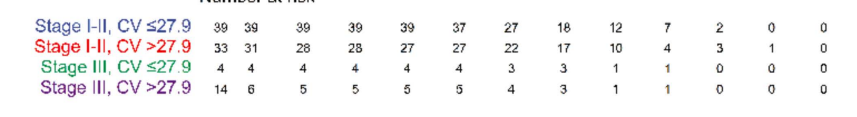

D

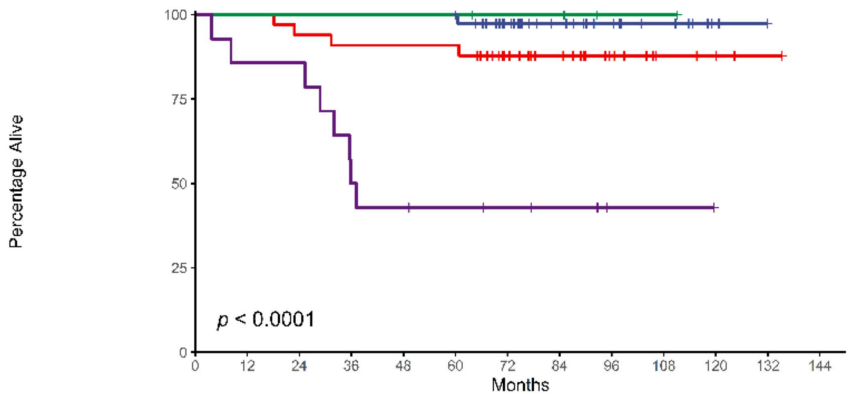

Number at risk

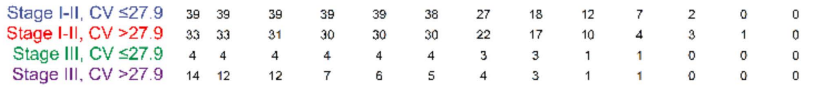

Figure 2. Kaplan-Meier estimates of progression-free survival (A,B) and overall survival (C,D) according to stage, and tumor $S_{U V}$ max to liver $S U V_{\text {mean }}$ ratio $\left(S_{\text {max }}-T L R\right)(A, C)$ or coefficient of variation (CV) (B,D). Global $p$ values are presented to compare progression-free survival and overall survival across groups. Tick marks represent censored data. 
Table 4. Patient characteristics for patients who received surgery as the primary treatment.

\begin{tabular}{|c|c|}
\hline Characteristic & Surgically Treated Patients $(n=77)$ \\
\hline Age, mean $\pm S D$, years & $57.4 \pm 8.4$ \\
\hline \multicolumn{2}{|l|}{ Sex, $n(\%)$} \\
\hline Male & $64(83.1)$ \\
\hline Female & $13(16.9)$ \\
\hline \multicolumn{2}{|l|}{ ECOG, $n(\%)$} \\
\hline 0 & $30(39.0)$ \\
\hline 1 & $47(61.0)$ \\
\hline \multicolumn{2}{|l|}{ Smoking history, $n(\%)$} \\
\hline Never or $\leq 10$ pack-years & $42(54.5)$ \\
\hline$>10$ pack-years & $35(45.5)$ \\
\hline \multicolumn{2}{|l|}{ Overall stage, $n(\%)$} \\
\hline Stage I & $58(75.3)$ \\
\hline Stage II & $8(10.4)$ \\
\hline Stage III & $11(14.3)$ \\
\hline \multicolumn{2}{|l|}{ Tumor stage, $n(\%)$} \\
\hline $\mathrm{T} 1$ & $16(20.8)$ \\
\hline $\mathrm{T} 2$ & $43(55.8)$ \\
\hline T3 & $7(9.1)$ \\
\hline $\mathrm{T} 4$ & $11(14.3)$ \\
\hline \multicolumn{2}{|l|}{ Nodal stage, $n(\%)$} \\
\hline No & $7(9.1)$ \\
\hline N1 & $64(83.1)$ \\
\hline N2 & $5(6.5)$ \\
\hline N3 & $1(1.3)$ \\
\hline \multicolumn{2}{|l|}{ Chemotherapy, $n(\%)$} \\
\hline Yes & $63(81.8)$ \\
\hline No & $14(18.2)$ \\
\hline $\mathrm{SUV}_{\max }-\mathrm{TLR}$, median (range) & $5.3(1.5-11.0)$ \\
\hline MTV, median (range), $\mathrm{mL}$ & $7.0(0.3-46.1)$ \\
\hline TLG-TLR, median (range) & $23.1(1.3-243.7)$ \\
\hline $\mathrm{CV}$, mean $\pm \mathrm{SD}, \%$ & $27.5 \pm 6.3$ \\
\hline
\end{tabular}

$\mathrm{SUV}_{\max }-\mathrm{TLR}$, tumor $\mathrm{SUV}_{\max }$ to liver $\mathrm{SUV}_{\text {mean }}$ ratio; MTV, metabolic tumor volume; TLG-TLR, tumor total lesion glycolysis to liver $\mathrm{SUV}_{\text {mean }}$ ratio; $\mathrm{CV}$, coefficient of variation.

Table 5. Univariate and multivariate analyses for progression-free survival for patients who received surgery as the primary treatment.

\begin{tabular}{|c|c|c|}
\hline Characteristic & Hazard Ratio (95\% CI) & $p^{*}$ \\
\hline \multicolumn{3}{|c|}{ Univariate Analysis } \\
\hline Age & & 0.22 \\
\hline$\leq 57$ years & & \\
\hline$>57$ years & $2.32(0.60-8.96)$ & \\
\hline Sex & & NR \\
\hline \multicolumn{3}{|l|}{ Male } \\
\hline Female & NR & \\
\hline ECOG & & 0.23 \\
\hline \multicolumn{3}{|l|}{0} \\
\hline 1 & $2.61(0.55-12.29)$ & \\
\hline Smoking history & & 0.36 \\
\hline \multicolumn{3}{|l|}{ Never or $\leq 10$ pack-years } \\
\hline$>10$ pack-years & $1.82(0.51-6.44)$ & \\
\hline Overall stage & & 0.012 \\
\hline \multicolumn{3}{|l|}{ I-II } \\
\hline III & $5.08(1.43-18.05)$ & \\
\hline Surgical approach & & 0.41 \\
\hline \multicolumn{3}{|l|}{ Open } \\
\hline Robotic & $0.59(0.17-2.09)$ & \\
\hline
\end{tabular}


Table 5. Cont.

\begin{tabular}{|c|c|c|}
\hline Characteristic & Hazard Ratio (95\% CI) & $p^{*}$ \\
\hline Resection margin & & 0.21 \\
\hline \multicolumn{3}{|l|}{ Negative } \\
\hline Positive & $2.23(0.64-7.69)$ & \\
\hline $\begin{array}{l}\text { Extracapsular spread } \\
\text { Negative }\end{array}$ & & 0.15 \\
\hline Positive & $3.10(0.66-14.58)$ & \\
\hline Lymphovascular invasion & & 0.01 \\
\hline \multicolumn{3}{|l|}{ Negative } \\
\hline Positive & $5.57(1.44-21.55)$ & \\
\hline Negative & & 0.14 \\
\hline Positive & $2.75(0.71-10.63)$ & \\
\hline \multicolumn{3}{|l|}{ Adjuvant therapy } \\
\hline Radiotherapy & $1.16(0.12-11.19)$ & 0.90 \\
\hline Chemoradiotherapy & $0.61(0.07-5.05)$ & 0.65 \\
\hline Chemotherapy & & 0.86 \\
\hline Yes & $0.87(0.18-4.08)$ & \\
\hline \multicolumn{3}{|l|}{ No } \\
\hline \multicolumn{2}{|l|}{$\leq 7.1$} & 0.003 \\
\hline$>7.1$ & 7.05 (1.97-25.17) & \\
\hline MTV & & 0.003 \\
\hline \multicolumn{3}{|l|}{$\leq 17.1 \mathrm{~mL}$} \\
\hline TLG-TLR & & 0.003 \\
\hline \multicolumn{3}{|l|}{$\leq 70.3$} \\
\hline$>70.3$ & 7.05 (1.97-25.17) & \\
\hline $\mathrm{CV}$ & & 0.007 \\
\hline \multicolumn{3}{|l|}{$\leq 29.8 \%$} \\
\hline$>29.8 \%$ & $8.31(1.76-39.20)$ & \\
\hline \multicolumn{3}{|c|}{ Multivariate analysis including overall stage, lymphovascular invasion, and SUV max $_{\text {-TLR (PFS model 1) }}$} \\
\hline Overall stage (I-II vs. III) & $4.74(1.20-18.68)$ & 0.03 \\
\hline Lymphovascular invasion (negative vs. positive) & $6.30(1.58-25.18)$ & 0.009 \\
\hline $\operatorname{SUV}_{\max }-\mathrm{TLR}(\leq 7.1$ vs. $>7.1)$ & $4.43(1.16-16.89)$ & 0.03 \\
\hline \multicolumn{3}{|c|}{ Multivariate analysis including overall stage, lymphovascular invasion, and MTV (PFS model 2) } \\
\hline Overall stage (I-II vs. III) & $4.05(0.98-16.75)$ & 0.054 \\
\hline Lymphovascular invasion (negative vs. positive) & $6.87(1.73-27.28)$ & 0.006 \\
\hline $\operatorname{MTV}(\leq 17.1$ vs. $>17.1 \mathrm{~mL})$ & $5.29(1.31-21.45)$ & 0.02 \\
\hline \multicolumn{3}{|c|}{ Multivariate analysis including overall stage, lymphovascular invasion, and TLG-TLR (PFS model 3) } \\
\hline Overall stage (I-II vs. III) & $4.74(1.20-18.68)$ & 0.03 \\
\hline Lymphovascular invasion (negative vs. positive) & $6.30(1.58-25.18)$ & 0.009 \\
\hline TLG-TLR $(\leq 70.3$ vs. $>70.3)$ & $4.43(1.16-16.89)$ & 0.03 \\
\hline \multicolumn{3}{|c|}{ Multivariate analysis including overall stage, lymphovascular invasion, and CV (PFS model 4) } \\
\hline Overall stage (I-II vs. III) & $6.74(1.69-26.88)$ & 0.007 \\
\hline Lymphovascular invasion (negative vs. positive) & $5.68(1.37-23.59)$ & 0.02 \\
\hline CV $(\leq 29.8$ vs. $>29.8 \%)$ & $6.40(1.33-30.79)$ & 0.02 \\
\hline
\end{tabular}

Hazard ratio is not reported for sex because of the low numbers of events. ${ }^{*}$ Wald test. CI, confidence interval; NR, not reported; $\mathrm{SUV}_{\text {max }}{ }^{-}$ TLR, tumor $\mathrm{SUV}_{\max }$ to liver $\mathrm{SUV}_{\text {mean }}$ ratio; MTV, metabolic tumor volume; TLG-TLR, tumor total lesion glycolysis to liver $S \mathrm{SV}_{\text {mean }}$ ratio;

$\mathrm{CV}$, coefficient of variation; PFS, progression-free survival. 
Table 6. Univariate and multivariate analyses for overall survival for patients who received surgery as the primary treatment.

\begin{tabular}{|c|c|c|}
\hline Characteristic & Hazard Ratio (95\% CI) & $p^{*}$ \\
\hline \multicolumn{3}{|c|}{ Univariate Analysis } \\
\hline Age & & 0.10 \\
\hline \multicolumn{3}{|l|}{$\leq 57$ years } \\
\hline$>57$ years & $6.05(0.73-50.25)$ & \\
\hline Sex & & NR \\
\hline \multicolumn{3}{|l|}{ Male } \\
\hline Female & NR & \\
\hline ECOG & & 0.21 \\
\hline \multicolumn{3}{|l|}{0} \\
\hline 1 & $3.89(0.47-32.31)$ & \\
\hline Smoking history & & 0.55 \\
\hline \multicolumn{3}{|l|}{ Never or $\leq 10$ pack-years } \\
\hline$>10$ pack-years & $1.58(0.35-7.07)$ & \\
\hline Overall stage & & 0.03 \\
\hline \multicolumn{3}{|l|}{ I-II } \\
\hline III & $5.34(1.19-23.94)$ & \\
\hline Surgical approach & & 0.22 \\
\hline \multicolumn{3}{|l|}{ Open } \\
\hline Robotic & $0.36(0.07-1.83)$ & \\
\hline Resection margin & & 0.16 \\
\hline \multicolumn{3}{|l|}{ Negative } \\
\hline Positive & $2.95(0.66-13.20)$ & \\
\hline Extracapsular spread & & 0.15 \\
\hline \multicolumn{3}{|l|}{ Negative } \\
\hline Positive & $4.68(0.56-38.85)$ & \\
\hline Lymphovascular invasion & & 0.04 \\
\hline \multicolumn{3}{|l|}{ Negative } \\
\hline Positive & $5.76(1.12-29.70)$ & \\
\hline Perineural invasion & & 0.28 \\
\hline \multicolumn{3}{|l|}{ Negative } \\
\hline Positive & $2.49(0.48-12.86)$ & \\
\hline Adjuvant therapy & & 0.15 \\
\hline \multicolumn{3}{|l|}{ Radiotherapy } \\
\hline Chemoradiotherapy & $0.33(0.07-1.49)$ & \\
\hline Chemotherapy & & 0.42 \\
\hline Yes & $0.51(0.10-2.62)$ & \\
\hline \multicolumn{3}{|l|}{ No } \\
\hline $\mathrm{SUV}_{\max }-\mathrm{TLR}$ & & 0.07 \\
\hline \multicolumn{3}{|l|}{$\leq 5.3$} \\
\hline$>5.3$ & $7.33(0.88-60.90)$ & \\
\hline MTV & & 0.07 \\
\hline \multicolumn{3}{|l|}{$\leq 15.9 \mathrm{~mL}$} \\
\hline$>15.9 \mathrm{~mL}$ & $4.08(0.91-18.26)$ & \\
\hline TLG-TLR & & 0.24 \\
\hline \multicolumn{3}{|l|}{$\leq 9.1$} \\
\hline$>9.1$ & $3.61(0.43-30.00)$ & \\
\hline $\mathrm{CV}$ & & 0.02 \\
\hline \multicolumn{3}{|l|}{$\leq 29.8 \%$} \\
\hline$>29.8 \%$ & 11.79 (1.42-98.02) & \\
\hline \multicolumn{3}{|c|}{ Multivariate analysis including overall stage, lymphovascular invasion, and CV } \\
\hline Overall stage (I-II vs. III) & $4.93(1.02-23.83)$ & 0.047 \\
\hline Lymphovascular invasion (negative vs. positive) & $4.55(0.85-24.33)$ & 0.08 \\
\hline CV $(\leq 29.8$ vs. $>29.8 \%)$ & $8.18(0.96-69.95)$ & 0.06 \\
\hline
\end{tabular}

Hazard ratio are not reported for sex and no adjuvant therapy because of the low numbers of events. ${ }^{*}$ Wald test. CI, confidence interval; NR, not reported; $\mathrm{SUV}_{\max }-\mathrm{TLR}$, tumor $\mathrm{SUV}_{\max }$ to liver $\mathrm{SUV}$ mean ratio; MTV, metabolic tumor volume; TLG-TLR, tumor total lesion glycolysis to liver $\mathrm{SUV}_{\text {mean }}$ ratio; $\mathrm{CV}$, coefficient of variation; OS, overall survival. 


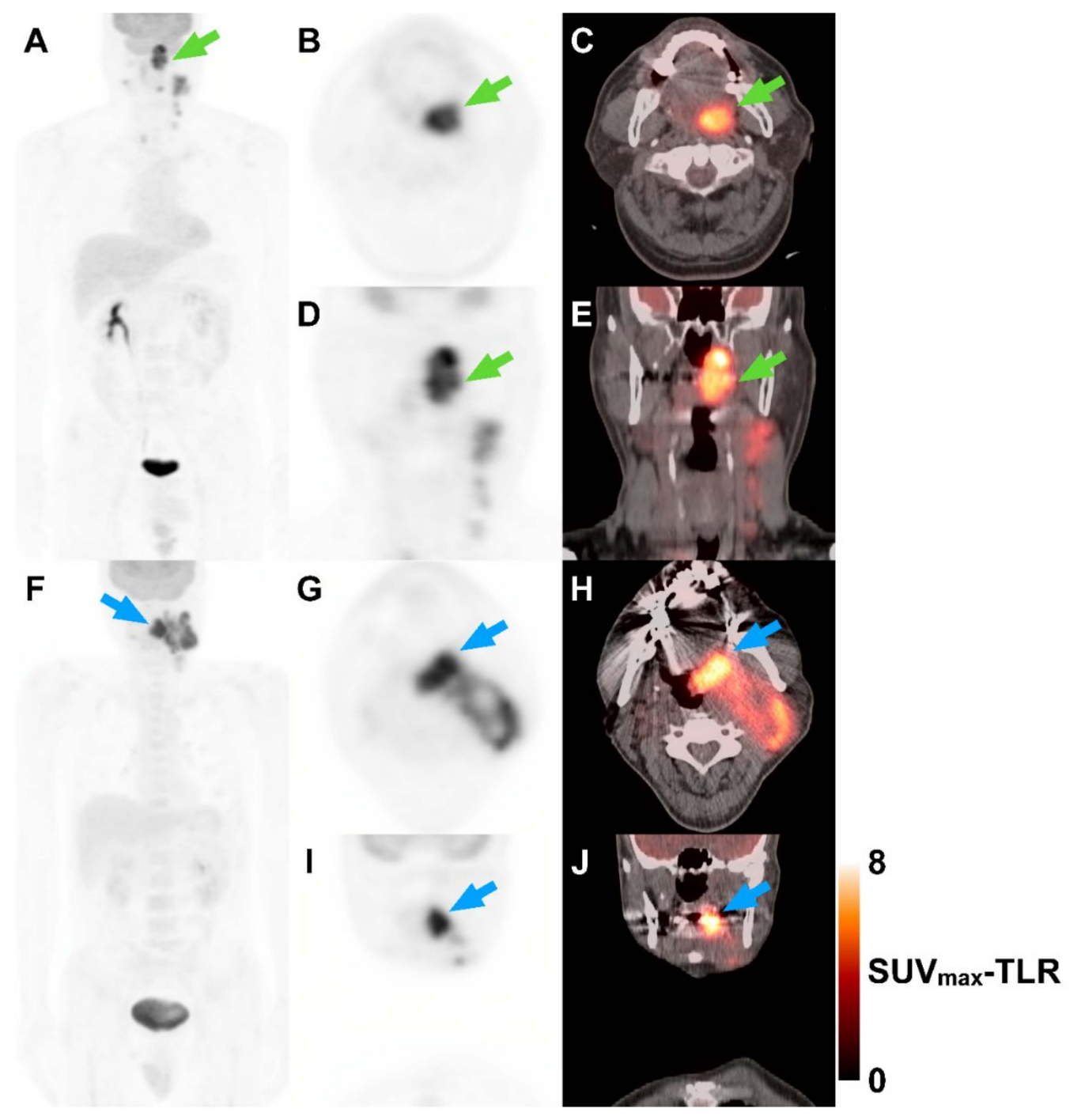

Figure 3. Two representative cases show that patients' outcomes could be estimated based on metabolic parameters. A 59-year-old man with HPV-related oropharyngeal cancer squamous cell carcinoma (OPSCC) (A-E). Maximum intensity projection (A), axial and coronal PET (B,D), and fused PET/CT (C,E) images demonstrate left tonsillar mass with bilateral cervical lymph node metastasis. The primary tumor shows heterogeneous FDG uptake (green arrows). The TNM designation is T4N2M0 with a prognostic grouping of stage III. Tumor SUV $\mathrm{max}_{\text {ax }}$ to liver $\mathrm{SUV}_{\text {mean }}$ ratio SUV $_{\max }{ }^{-}$ TLR) and coefficient of variation (CV) are 8.0 and $34.5 \%$, respectively. The patient progressed after 11.6 months and died after 28.8 months. Another 59-year-old man with HPV-related OPSCC (F-J). Maximum intensity projection $(\mathbf{F})$, axial and coronal PET $(\mathbf{G}, \mathbf{I})$, and fused PET/CT $(\mathbf{H}, \mathbf{J})$ images reveal left tonsillar mass with bilateral cervical lymph node metastasis. The primary tumor shows relatively homogeneous FDG uptake (blue arrows). The TNM designation is T4N3M0 with a prognostic grouping of stage III. SUV $\max _{-T L R}$ and CV are 7.0 and $27.9 \%$, respectively. The patient has not progressed or died during the 63.8-month follow-up. 
A

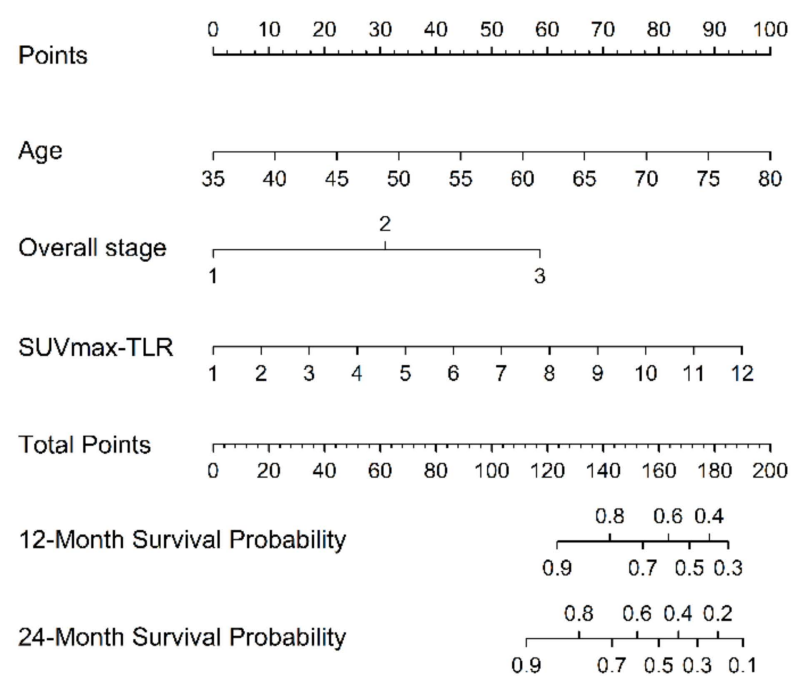

C

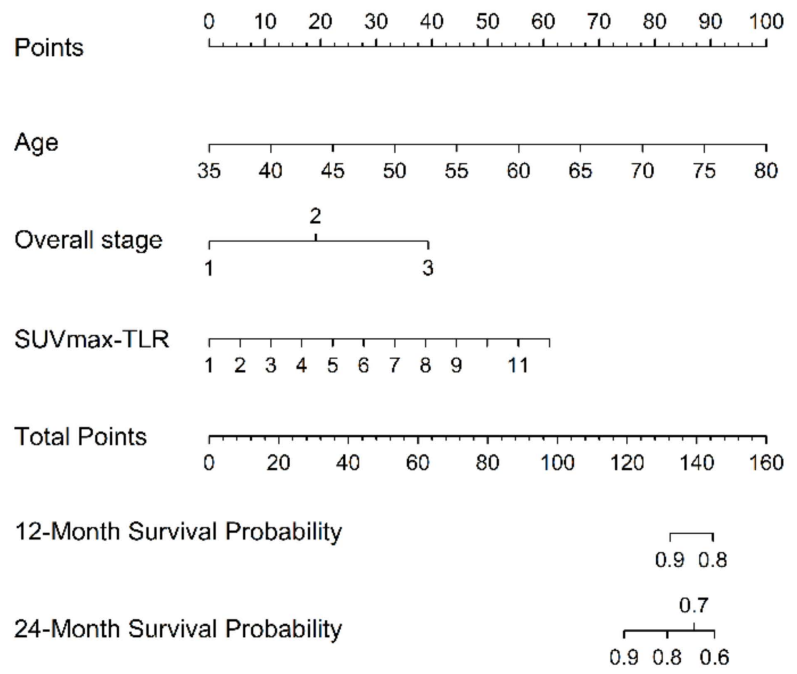

B

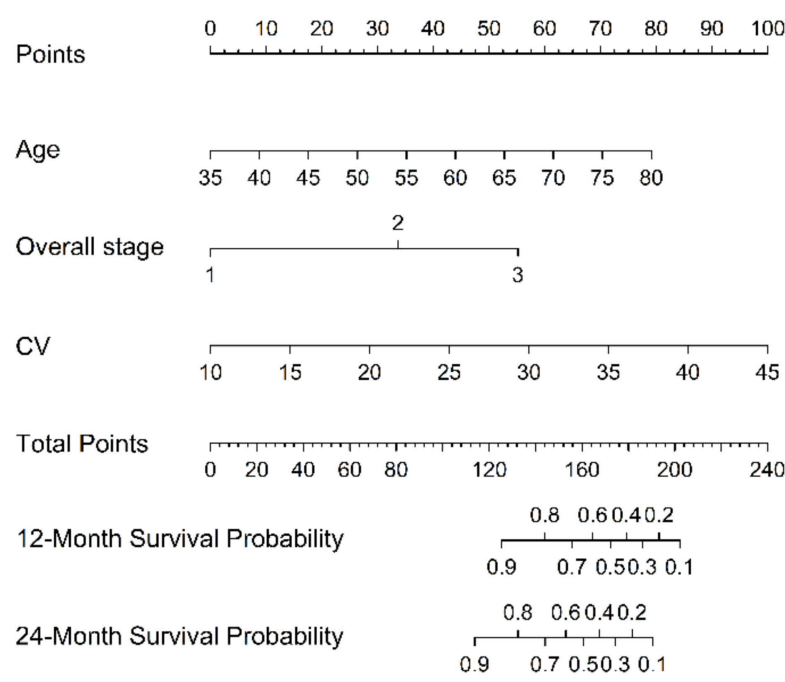

D

Points

$\begin{array}{lllllllllll}0 & 10 & 20 & 30 & 40 & 50 & 60 & 70 & 80 & 90 & 100\end{array}$

Age

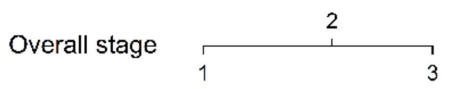

CV

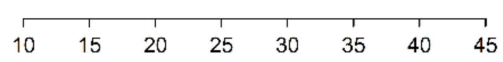

Total Points

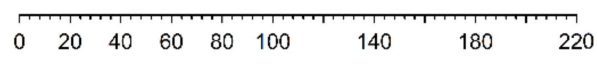

12-Month Survival Probability

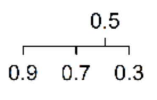

24-Month Survival Probability

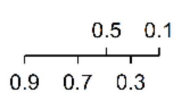

Figure 4. Nomograms predicting the 1- and 2-year progression-free (A,B) and overall $(\mathbf{C}, \mathbf{D})$ survival rates. To calculate predicted survival, a straight line is drawn up to the row labeled "Points" to determine the corresponding points for each factor. The total points projected on the bottom scales show the probabilities of 1- and 2-year progression-free survival or overall survival. SUV $\max -T L R$, tumor $\mathrm{SUV}_{\max }$ to liver $\mathrm{SUV}_{\text {mean }}$ ratio; $\mathrm{CV}$, coefficient of variation.

\section{Discussion}

Our results show that metabolic features on baseline F-18 FDG PET/CT, such as higher tumor glucose metabolism derived from $\mathrm{SUV}_{\max }-\mathrm{TLR}$, and increased intratumoral heterogeneity inferred from $\mathrm{CV}$, were associated with poorer PFS and OS after adjusting other clinical factors in patients with nonmetastatic HPV-related OPSCC using the 8th TNM staging system. We evaluated the prognostic value of a number of metabolic parameters on baseline F-18 FDG PET/CT and compared these metabolic parameters with known prognostic indicators, such as stage and smoking history. Primary treatment and volumetric parameters such as MTV and TLG on F-18 FDG PET/CT were not significant prognostic indicators in predicting PFS and OS in multivariate analysis. In surgically treated patients, we also found that metabolic parameters were significant prognostic indicators. Currently, the TNM staging system is the most important prognostic indicator. However, even after the revision to the 8th TNM staging system, the TNM staging system does not always provide accurate prognostic prediction. This study shows the potential role of metabolic 
parameters on F-18 FDG PET/CT in identifying different risks in patients with HPV-related OPSCC and may aid in future de-escalation trials by revealing low-risk patients.

F-18 FDG PET/CT has been widely used in diagnosis, staging, monitoring response to therapy, and prognostication of various tumors [31,32]. Increased glycolysis and metabolic heterogeneity are associated with poorer outcomes [33,34]. A number of studies have investigated the association between $\mathrm{SUV}_{\max }, \mathrm{SUV}_{\text {peak }}, \mathrm{MTV}$, TLG, or intratumoral heterogeneity of primary tumor and lymph node on baseline F-18 FDG PET/CT with OS, disease-free survival (DFS), or event-free survival (EFS) in patients with HPV-related OPSCC, and the results were inconclusive. Most studies assessed the stage using the 7th TNM staging system. Higher $\mathrm{SUV}_{\max }, \mathrm{MTV}, \mathrm{TLG}$, and intratumoral heterogeneity of the primary tumor or lymph node were reported as significant prognosticators of OS, DFS, or EFS. Kim et al. [25] evaluated the prognostic roles of F-18 FDG PET/CT in 86 patients with stage II-IV HPV-related OPSCC. They found that higher nodal $\mathrm{MTV}_{40 \%}$ and $\mathrm{MTV}_{40 \%}$ or TLG of combined primary tumor and node are associated with worse DFS. The patients were treated with surgery followed by radiotherapy or chemoradiotherapy. Mena et al. [26] revealed that higher $\mathrm{SUV}_{\max }$ or $\mathrm{MTV}_{50 \%}$ with increased tumoral heterogeneity derived from a cumulative SUV-volume histogram curve is correlated with poorer EFS in 105 patients with stage I-IV HPV-related OPSCC. The treatment modalities including chemoradiotherapy and surgery, followed by chemoradiotherapy or radiotherapy, were heterogeneous in this group of patients. Floberg et al. [35] explored the significance of $\mathrm{MTV}_{50 \%}$ of the primary tumor and lymph node in 153 patients with stage I-III HPV-related OPSCC using the 8th TNM staging system. These patients were treated with radiotherapy or surgery, followed by radiotherapy combined with/without chemotherapy. Higher MTV is associated with worse OS, EFS, and distant metastasis-free survival. However, some studies reported no significant association between metabolic parameters, such as $\mathrm{SUV}_{\max }$, $\mathrm{SUV}_{\text {peak }}, \mathrm{MTV}_{33 \%}$, or $\mathrm{MTV}_{41 \%}$, and TLG of the primary tumor in predicting OS, DFS, or local control in patients with stage I-IV HPV-related OPSCC $[27,28]$. These studies also included patients with HPV-unrelated OPSCC or other head and neck cancers, and the patients were treated with chemoradiotherapy or radiotherapy.

The heterogeneous results may be attributed to the differences in the patient population in terms of prognosis, primary treatment, and quantification of metabolic parameters. As stated earlier, HPV-related OPSCC emerged as a distinct disease with a favorable prognosis. Current treatment is intended for tobacco-related head and neck cancers and it may be more intensive than it needed to be cured. In this regard, various approaches have been attempted to de-escalate current treatment while maintaining the current cure rate. An earlier study revealed that patients with N2c HPV-related OPSCC using the 6th TNM staging system show impaired distant control, suggesting a subset of patients with HPV-related OPSCC carry a poorer prognosis [36]. T4 or N3 disease and tobacco exposure for more than 10 pack-years are known to be associated with higher risk. Two randomized trials have assessed radiotherapy with cisplatin or cetuximab, a less toxic alternative to cisplatin, in patients with HPV-related OPSCC and found a significant benefit in OS and PFS in favor of cisplatin. In the subgroup analysis which only includes low-risk patients (T1-T3, N0-N2 (using the 7th TNM staging system), and non-smokers), the survival benefit in favor of cisplatin was still observed [37,38]. The above findings prompt the need for well-defined, low-risk patients for future de-escalation trials.

SUV has been widely used to quantify PET images and regarded as a robust and reproducible parameter for analyzing patients. However, various sources of bias can affect SUV measurement. Patient weight, blood glucose level, administered activity, the time elapsed from the injection, and imaging protocol may have contributed to the bias [39-41]. MTV and TLG have also received considerable attention to quantify various tumors, and are assumed to be stronger predictors than $S_{U V}$ max in head and neck cancer [42]. However, no consensus has been reached regarding threshold methods and their prognostic significance $[43,44]$. To mitigate these methodological differences, we adopted the tumor to liver ratio for $\mathrm{SUV}_{\max }$ and TLG and set the threshold for MTV based on liver uptake [29,45]. 
Efforts have been made to improve risk stratification in patients with HPV-related OPSCC. Most studies have focused on patients managed with definitive chemoradiotherapy and showed that prognostication can be improved by incorporating advanced $\mathrm{T}$ stage and $\mathrm{N}$ stage based on nasopharyngeal cancer $\mathrm{N}$ categories [10,12,36,46,47]. A study explored surgically treated patients and revealed that a composite risk stratification system which includes pathologic adverse features such as lymphovascular invasion, surgical margins, extranodal extension, advanced $\mathrm{T}$ stage, and the number of metastatic lymph nodes improve prognostication [48]. However, these models need further validation. Further refinement of prognostication for patients with HPV-OPSCC will provide appropriate treatment, and incorporation of metabolic parameters could be beneficial for improvement of prognostication.

This study has some limitations. The design is retrospective, and a small number of patients were analyzed. Moreover, primary treatment was varied among the patients, and most patients were primarily treated with surgery. Thus, inherent biases may have affected the results. Further validation is warranted in a large number of patients with a prospective design to assess potential prognostic roles of metabolic parameters.

\section{Conclusions}

We showed that higher tumor glucose uptake and its heterogeneity on F-18 FDG $\mathrm{PET} / \mathrm{CT}$ were associated with poorer PFS and OS in patients with nonmetastatic HPVrelated OPSCC. These findings imply that the metabolic parameters on F-18 FDG PET/CT may aid in better stratification along with the current 8th TNM staging system in patients with HPV-related OPSCC and in designing future de-escalation trials.

Author Contributions: Conceptualization, H.C. and W.J.K.; methodology, H.C. and W.J.K.; software, H.C., K.J. and W.J.K.; validation, H.C., K.J. and W.J.K.; formal analysis, H.C., K.J. and W.J.K.; investigation, H.C. and W.J.K.; resources, H.C. and W.J.K.; data curation, H.C., S.K. and Y.H.J.; writing-original draft preparation, H.C., S.K. and K.J.; writing-review and editing, Y.H.J. and W.J.K.; visualization, H.C. and K.J.; supervision, W.J.K.; project administration, W.J.K.; funding acquisition, W.J.K. All authors have read and agreed to the published version of the manuscript.

Funding: This research was funded by the Korea government (MSIT), grant number 2020M2D9A1093991.

Institutional Review Board Statement: The study was conducted according to the guidelines of the Declaration of Helsinki and approved by the Institutional Review Board of Severance Hospital (4-2021-0397, May 2021).

Informed Consent Statement: Patient consent was waived due to the retrospective nature of the study.

Data Availability Statement: The data analyzed in the current study are available from the corresponding author on reasonable request.

Conflicts of Interest: The authors declare no conflict of interest.

\section{References}

1. Bray, F.; Ferlay, J.; Soerjomataram, I.; Siegel, R.L.; Torre, L.A.; Jemal, A. Global cancer statistics 2018: GLOBOCAN estimates of incidence and mortality worldwide for 36 cancers in 185 countries. CA Cancer J. Clin. 2018, 68, 394-424. [CrossRef] [PubMed]

2. Chaturvedi, A.K.; Engels, E.A.; Pfeiffer, R.M.; Hernandez, B.Y.; Xiao, W.; Kim, E.; Jiang, B.; Goodman, M.T.; Sibug-Saber, M.; Cozen, W.; et al. Human papillomavirus and rising oropharyngeal cancer incidence in the United States. J. Clin. Oncol. 2011, 29, 4294-4301. [CrossRef] [PubMed]

3. Gillison, M.L.; Chaturvedi, A.K.; Anderson, W.F.; Fakhry, C. Epidemiology of Human Papillomavirus-Positive Head and Neck Squamous Cell Carcinoma. J. Clin. Oncol. 2015, 33, 3235-3242. [CrossRef] [PubMed]

4. You, E.L.; Henry, M.; Zeitouni, A.G. Human papillomavirus-associated oropharyngeal cancer: Review of current evidence and management. Curr. Oncol. 2019, 26, 119-123. [CrossRef] [PubMed]

5. Pytynia, K.B.; Dahlstrom, K.R.; Sturgis, E.M. Epidemiology of HPV-associated oropharyngeal cancer. Oral Oncol. 2014, 50, 380-386. [CrossRef] 
6. Mehanna, H.; Beech, T.; Nicholson, T.; El-Hariry, I.; McConkey, C.; Paleri, V.; Roberts, S. Prevalence of human papillomavirus in oropharyngeal and nonoropharyngeal head and neck cancer-Systematic review and meta-analysis of trends by time and region. Head Neck 2013, 35, 747-755. [CrossRef]

7. Fakhry, C.; Gillison, M.L. Clinical implications of human papillomavirus in head and neck cancers. J. Clin. Oncol. 2006, 24, 2606-2611. [CrossRef]

8. Leemans, C.R.; Snijders, P.J.F.; Brakenhoff, R.H. The molecular landscape of head and neck cancer. Nat. Rev. Cancer 2018, 18, 269-282. [CrossRef]

9. Taberna, M.; Mena, M.; Pavon, M.A.; Alemany, L.; Gillison, M.L.; Mesia, R. Human papillomavirus-related oropharyngeal cancer. Ann. Oncol. 2017, 28, 2386-2398. [CrossRef]

10. Ang, K.K.; Harris, J.; Wheeler, R.; Weber, R.; Rosenthal, D.I.; Nguyen-Tan, P.F.; Westra, W.H.; Chung, C.H.; Jordan, R.C.; Lu, C.; et al. Human papillomavirus and survival of patients with oropharyngeal cancer. N. Engl. J. Med. 2010, 363, 24-35. [CrossRef]

11. Nguyen-Tan, P.F.; Zhang, Q.; Ang, K.K.; Weber, R.S.; Rosenthal, D.I.; Soulieres, D.; Kim, H.; Silverman, C.; Raben, A.; Galloway, T.J.; et al. Randomized phase III trial to test accelerated versus standard fractionation in combination with concurrent cisplatin for head and neck carcinomas in the Radiation Therapy Oncology Group 0129 trial: Long-term report of efficacy and toxicity. J. Clin. Oncol. 2014, 32, 3858-3866. [CrossRef]

12. O'Sullivan, B.; Huang, S.H.; Su, J.; Garden, A.S.; Sturgis, E.M.; Dahlstrom, K.; Lee, N.; Riaz, N.; Pei, X.; Koyfman, S.A.; et al. Development and validation of a staging system for HPV-related oropharyngeal cancer by the International Collaboration on Oropharyngeal cancer Network for Staging (ICON-S): A multicentre cohort study. Lancet Oncol. 2016, 17, 440-451. [CrossRef]

13. Brierley, J.; Gospodarowicz, M.K.; Wittekind, C. TNM Classification of Malignant Tumours, 8th ed.; John Wiley \& Sons, Inc.: Hoboken, NJ, USA, 2017.

14. Fakhry, C.; Westra, W.H.; Li, S.; Cmelak, A.; Ridge, J.A.; Pinto, H.; Forastiere, A.; Gillison, M.L. Improved survival of patients with human papillomavirus-positive head and neck squamous cell carcinoma in a prospective clinical trial. J. Natl. Cancer Inst. 2008, 100, 261-269. [CrossRef]

15. Adelstein, D.J.; Ridge, J.A.; Brizel, D.M.; Holsinger, F.C.; Haughey, B.H.; O’Sullivan, B.; Genden, E.M.; Beitler, J.J.; Weinstein, G.S.; Quon, H.; et al. Transoral resection of pharyngeal cancer: Summary of a National Cancer Institute Head and Neck Cancer Steering Committee Clinical Trials Planning Meeting, 6-7 November 2011, Arlington, Virginia. Head Neck 2012, 34, 1681-1703. [CrossRef]

16. Cracchiolo, J.R.; Baxi, S.S.; Morris, L.G.; Ganly, I.; Patel, S.G.; Cohen, M.A.; Roman, B.R. Increase in primary surgical treatment of $\mathrm{T} 1$ and T2 oropharyngeal squamous cell carcinoma and rates of adverse pathologic features: National Cancer Data Base. Cancer 2016, 122, 1523-1532. [CrossRef]

17. Gregoire, V.; Lefebvre, J.L.; Licitra, L.; Felip, E.; Group, E.-E.-E.G.W. Squamous cell carcinoma of the head and neck: EHNSESMO-ESTRO Clinical Practice Guidelines for diagnosis, treatment and follow-up. Ann. Oncol. 2010, 21 (Suppl. S5), v184-v186. [CrossRef]

18. Marur, S.; D'Souza, G.; Westra, W.H.; Forastiere, A.A. HPV-associated head and neck cancer: A virus-related cancer epidemic. Lancet Oncol. 2010, 11, 781-789. [CrossRef]

19. Hoxbroe Michaelsen, S.; Gronhoj, C.; Hoxbroe Michaelsen, J.; Friborg, J.; von Buchwald, C. Quality of life in survivors of oropharyngeal cancer: A systematic review and meta-analysis of 1366 patients. Eur. J. Cancer 2017, 78, 91-102. [CrossRef]

20. Quon, H.; Forastiere, A.A. Controversies in treatment deintensification of human papillomavirus-associated oropharyngeal carcinomas: Should we, how should we, and for whom? J. Clin. Oncol. 2013, 31, 520-522. [CrossRef]

21. Adelstein, D.J.; Ismaila, N.; Ku, J.A.; Burtness, B.; Swiecicki, P.L.; Mell, L.; Beitler, J.J.; Gross, N.; Jones, C.U.; Kaufman, M.; et al. Role of Treatment Deintensification in the Management of p16+ Oropharyngeal Cancer: ASCO Provisional Clinical Opinion. J. Clin. Oncol. 2019, 37, 1578-1589. [CrossRef]

22. Subramaniam, R.M.; Alluri, K.C.; Tahari, A.K.; Aygun, N.; Quon, H. PET/CT imaging and human papilloma virus-positive oropharyngeal squamous cell cancer: Evolving clinical imaging paradigm. J. Nucl. Med. 2014, 55, 431-438. [CrossRef]

23. Plaxton, N.A.; Brandon, D.C.; Corey, A.S.; Harrison, C.E.; Karagulle Kendi, A.T.; Halkar, R.K.; Barron, B.J. Characteristics and Limitations of FDG PET/CT for Imaging of Squamous Cell Carcinoma of the Head and Neck: A Comprehensive Review of Anatomy, Metastatic Pathways, and Image Findings. AJR Am. J. Roentgenol. 2015, 205, W519-W531. [CrossRef]

24. Taghipour, M.; Sheikhbahaei, S.; Marashdeh, W.; Solnes, L.; Kiess, A.; Subramaniam, R.M. Use of 18F-Fludeoxyglucose-Positron Emission Tomography/Computed Tomography for Patient Management and Outcome in Oropharyngeal Squamous Cell Carcinoma: A Review. JAMA Otolaryngol. Head Neck Surg. 2016, 142, 79-85. [CrossRef]

25. Kim, K.H.; Lee, J.; Chang, J.S.; Lee, C.G.; Yun, M.; Choi, E.C.; Kim, S.H.; Keum, K.C. Prognostic value of FDG-PET volumetric parameters in patients with p16-positive oropharyngeal squamous cell carcinoma who received curative resection followed by postoperative radiotherapy or chemoradiotherapy. Head Neck 2016, 38, 1515-1524. [CrossRef]

26. Mena, E.; Taghipour, M.; Sheikhbahaei, S.; Jha, A.K.; Rahmim, A.; Solnes, L.; Subramaniam, R.M. Value of Intratumoral Metabolic Heterogeneity and Quantitative 18F-FDG PET/CT Parameters to Predict Prognosis in Patients With HPV-Positive Primary Oropharyngeal Squamous Cell Carcinoma. Clin. Nucl. Med. 2017, 42, e227-e234. [CrossRef]

27. Gouw, Z.A.R.; La Fontaine, M.D.; van Kranen, S.; van de Kamer, J.B.; Vogel, W.V.; van Werkhoven, E.; Sonke, J.J.; Al-Mamgani, A. The Prognostic Value of Baseline 18F-FDG PET/CT in Human Papillomavirus-Positive Versus Human Papillomavirus-Negative Patients with Oropharyngeal Cancer. Clin. Nucl. Med. 2019, 44, e323-e328. [CrossRef] 
28. Moan, J.M.; Amdal, C.D.; Malinen, E.; Svestad, J.G.; Bogsrud, T.V.; Dale, E. The prognostic role of 18F-fluorodeoxyglucose PET in head and neck cancer depends on HPV status. Radiother. Oncol. 2019, 140, 54-61. [CrossRef]

29. Wahl, R.L.; Jacene, H.; Kasamon, Y.; Lodge, M.A. From RECIST to PERCIST: Evolving Considerations for PET response criteria in solid tumors. J. Nucl. Med. 2009, 50 (Suppl. S1), 122S-150S. [CrossRef]

30. Delgado, J.; Pereira, A.; Villamor, N.; Lopez-Guillermo, A.; Rozman, C. Survival analysis in hematologic malignancies: Recommendations for clinicians. Haematologica 2014, 99, 1410-1420. [CrossRef]

31. Kostakoglu, L.; Agress, H., Jr.; Goldsmith, S.J. Clinical role of FDG PET in evaluation of cancer patients. Radiographics 2003, 23, 315-340. [CrossRef]

32. Boellaard, R.; Delgado-Bolton, R.; Oyen, W.J.; Giammarile, F.; Tatsch, K.; Eschner, W.; Verzijlbergen, F.J.; Barrington, S.F.; Pike, L.C.; Weber, W.A.; et al. FDG PET/CT: EANM procedure guidelines for tumour imaging: Version 2.0. Eur. J. Nucl. Med. Mol. Imaging 2015, 42, 328-354. [CrossRef] [PubMed]

33. Gatenby, R.A.; Gillies, R.J. Why do cancers have high aerobic glycolysis? Nat. Rev. Cancer 2004, 4, 891-899. [CrossRef] [PubMed]

34. Yoshida, G.J. Metabolic reprogramming: The emerging concept and associated therapeutic strategies. J. Exp. Clin. Cancer Res. 2015, 34, 111. [CrossRef] [PubMed]

35. Floberg, J.M.; DeWees, T.A.; Chin, R.I.; Garsa, A.A.; Dehdashti, F.; Nussenbaum, B.; Oppelt, P.J.; Adkins, D.R.; Gay, H.A.; Thorstad, W.L. Pretreatment metabolic tumor volume as a prognostic factor in HPV-associated oropharyngeal cancer in the context of AJCC 8th edition staging. Head Neck 2018, 40, 2280-2287. [CrossRef]

36. O'Sullivan, B.; Huang, S.H.; Siu, L.L.; Waldron, J.; Zhao, H.; Perez-Ordonez, B.; Weinreb, I.; Kim, J.; Ringash, J.; Bayley, A.; et al . Deintensification candidate subgroups in human papillomavirus-related oropharyngeal cancer according to minimal risk of distant metastasis. J. Clin. Oncol. 2013, 31, 543-550. [CrossRef]

37. Gillison, M.L.; Trotti, A.M.; Harris, J.; Eisbruch, A.; Harari, P.M.; Adelstein, D.J.; Jordan, R.C.K.; Zhao, W.; Sturgis, E.M.; Burtness, B.; et al. Radiotherapy plus cetuximab or cisplatin in human papillomavirus-positive oropharyngeal cancer (NRG Oncology RTOG 1016): A randomised, multicentre, non-inferiority trial. Lancet 2019, 393, 40-50. [CrossRef]

38. Mehanna, H.; Robinson, M.; Hartley, A.; Kong, A.; Foran, B.; Fulton-Lieuw, T.; Dalby, M.; Mistry, P.; Sen, M.; O’Toole, L.; et al. Radiotherapy plus cisplatin or cetuximab in low-risk human papillomavirus-positive oropharyngeal cancer (De-ESCALaTE HPV): An open-label randomised controlled phase 3 trial. Lancet 2019, 393, 51-60. [CrossRef]

39. Boellaard, R. Standards for PET image acquisition and quantitative data analysis. J. Nucl. Med. 2009, 50 (Suppl. S1), 11S-20S. [CrossRef]

40. Westerterp, M.; Pruim, J.; Oyen, W.; Hoekstra, O.; Paans, A.; Visser, E.; van Lanschot, J.; Sloof, G.; Boellaard, R. Quantification of FDG PET studies using standardised uptake values in multi-centre trials: Effects of image reconstruction, resolution and ROI definition parameters. Eur. J. Nucl. Med. Mol. Imaging 2007, 34, 392-404. [CrossRef]

41. Adams, M.C.; Turkington, T.G.; Wilson, J.M.; Wong, T.Z. A systematic review of the factors affecting accuracy of SUV measurements. AJR Am. J. Roentgenol. 2010, 195, 310-320. [CrossRef]

42. Pak, K.; Cheon, G.J.; Nam, H.Y.; Kim, S.J.; Kang, K.W.; Chung, J.K.; Kim, E.E.; Lee, D.S. Prognostic value of metabolic tumor volume and total lesion glycolysis in head and neck cancer: A systematic review and meta-analysis. J. Nucl. Med. 2014, 55, 884-890. [CrossRef]

43. Im, H.J.; Bradshaw, T.; Solaiyappan, M.; Cho, S.Y. Current Methods to Define Metabolic Tumor Volume in Positron Emission Tomography: Which One is Better? Nucl. Med. Mol. Imaging 2018, 52, 5-15. [CrossRef]

44. Schoder, H.; Moskowitz, C. Metabolic Tumor Volume in Lymphoma: Hype or Hope? J. Clin. Oncol. 2016, 34, 3591-3594. [CrossRef]

45. Hofheinz, F.; Butof, R.; Apostolova, I.; Zophel, K.; Steffen, I.G.; Amthauer, H.; Kotzerke, J.; Baumann, M.; van den Hoff, J. An investigation of the relation between tumor-to-liver ratio (TLR) and tumor-to-blood standard uptake ratio (SUR) in oncological FDG PET. EJNMMI Res. 2016, 6, 19. [CrossRef]

46. Huang, S.H.; Xu, W.; Waldron, J.; Siu, L.; Shen, X.; Tong, L.; Ringash, J.; Bayley, A.; Kim, J.; Hope, A.; et al. Refining American Joint Committee on Cancer/Union for International Cancer Control TNM stage and prognostic groups for human papillomavirusrelated oropharyngeal carcinomas. J. Clin. Oncol. 2015, 33, 836-845. [CrossRef]

47. Dahlstrom, K.R.; Garden, A.S.; William, W.N., Jr.; Lim, M.Y.; Sturgis, E.M. Proposed Staging System for Patients With HPV-Related Oropharyngeal Cancer Based on Nasopharyngeal Cancer N Categories. J. Clin. Oncol. 2016, 34, 1848-1854. [CrossRef]

48. Cramer, J.D.; Dundar, Y.; Hotaling, J.; Raza, S.N.; Lin, H.S. Development and Assessment of a Novel Composite Pathologic Risk Stratification for Surgically Resected Human Papillomavirus-Associated Oropharyngeal Cancer. JAMA Otolaryngol. Head Neck Surg. 2019, 145, 1105-1114. [CrossRef] 